\title{
Concepto de Salud
}

\section{Francisco Javier Yuste Grijalba}

No es tiempo perdido recordar que la mayor preocupación de los interesados en la salud pública ha de centrarse en la salud y no en la enfermedad, por lo que no parece descabellado acercarnos al estudio del concepto de salud pública a través de la definición pura y simple de salud.

Las distintas concepciones - sincrónicas y diacrónicas- de salud no son categoría desconexas entre sí, más bien son puntos de vista de distintos observadores sobre la misma realidad. Si iniciamos nuestro recorrido por los conceptos desde su mismo origen, encontraremos como en el pensamiento grecorromano está implícito el futuro desarrollo de toda la realidad que llamamos salud. Higeia, diosa griega de la salud, armonía entre contrarios, cede su puesto a Sanitas en la hagiografía latina, pero si Higeia era teoria, Sanitas en praxis saneamiento, obras públicas, solución práctica de problemas.

Desde su etiología, la salud ha sido abordada en el campo médico como una realidad somática, como una realidad síquica y como una totalidad a la vez física, síquica y social. ${ }^{1}$

Salud en la concepción fisiológica es la ausencia de enfermedad en los individuos, el bienestar del cuerpo, y la enfermedad el proceso que altera este bienestar. La concepción somática de salud ha dominado la historia de la medicina desde sus orígenes hasta el siglo pasado, cuando se toma conciencia de que la salud no puede restringirse a lo orgánico y que debe incluir lo síquico, no sólo en cuanto defecto de la sique sino también como influencia del siquismo en la patología o estabilidad fisiológica.

Un paso adelante en las concepciones médicas de salud está representada por la concepción sanitaria que sin dejar de ser médica, dirige su mirada a la colectividad comprendiendo la influencia de los factores sociales en su mantenimiento y sostenimiento. ${ }^{2}$ Sin dejar de ser médica, esta concepción se caracteriza por su visión positiva de la salud - se trata de aumentarla mediante actividades que lo consiguen - 3 preventiva - se trata de impedir su pérdida por éncima de todo - y global —poniendo en relación la salud individual, la colectiva y la ambiental - lo que incluye evidentemente lo síquico.

Algo obvio, que la salud es un bien, no parece reconocido como derecho "hasta que existe una conciencia social por parte de los ciudadanos que reclama una acción en este terreno", es decir, hasta que la revolución burguesa y la proletaria han proclamado

(1) Sigo en estos párrafos a R. Moragas en Enfoque Sociologico de diversas concepciones de Salud, Papers. Revista de Sociologia (5). 1976. pp. 31 y ss.

(2) Lo que conects esta situación con los sociólogos.

(3) Vivienda, trabajo, educación, ocio, etc., como veremos después.

(4) R. Moragas, o.c. pp. 45 y ss. 
los derechos del hombre y del ciudadano. Históricamente Bismarck y Beveridge personifican estos transcendentales momentos en la Europa Occidental. ${ }^{5}$ La salud como bien que debe ser tutelado por el ordenamiento jurídico, ya no es un modelo médico sino legal que nos acerca a las concepciones económica y sociológica.

La salud es un bien que puede valorarse en términos económicos, tanto como potencialidad productiva como en las necesidades financieras para su mantenimiento. La importancia económica de la salud no ha sido todavía bien comprendida, dando importancia mayor a los costes de la curación de su pérdida que al precio de su mantenimiento. ${ }^{6}$

Hoy en día, la salud no se comprende sino como fenómeno social: generada para y en una sociedad, hace referencia en su nivel a una sociedad determinada, a su cultura, a su grado de desarrollo económico, a su organización política y sanitaria, en suma, la salud es un producto social. A partir de esta concepción se abre paso una nueva perspectiva que se ha llamado "era de la salud entendida como ciencia política". ${ }^{7}$ En esta nueva era lo importante será dar paso a la gente, es decir, a la población entera y reconocer que las causas de la mala salud están en los sufrimientos humanos básicos que requieren esfuerzos intersectoriales para aliviarlos.

La ciencia nueva requerirá información clara de los objetivos, fines y funciones que permita determinar las aptitudes y conocimientos teóricos indispensables en términos comprensibles para la población, los políticos y los profesionales. También se requeriran dirigentes intrépidos que sean capaces de romper los límites rutinarios en los que estamos metidos.

Al final de todas estas concepciones, como resumen de ellas, una concepción ideal de salud que nos lleva de nuevo a la idea de armonía con la que empezábamos nuestro recorrido: "la salud como estado de completo bienestar físico, mental y social y no únicamente la ausencia de enfermedad o dolencia" tal y como se define en la Constitución de la OMS en 1960. ¿Es posible conseguir tal tipo de salud? Parece claro que tal situación es relativa, no tanto un estado cuanto una tendencia a un límite en el que se confunden calidad de vida, bienestar, felicidad y máxima vitalidad. Lo ideal en esta definición ha suscitado alguna crítica, por quien se siente poco motivado para la acción por ella al confundir lo que es guia y tensión con lo que es inalcanzabilidad. Otra definición asimismo ideal, ha sido emitida en ambientes catalanes poniendo en relación las ideas de armonía, bienestar y calidad de vida con los componentes físico-síquico y social de la salud, la siguiente: "la salud es la forma de vivir autónoma, solidaria y profundamente alegre". ${ }^{8}$

\section{Concepto de Salud Pública}

La impronta de la eficacia de las obras públicas romanas, junto a la penuria

(5) En España, la salud como derecho de todos, sólo es consagrado en la Constitución del 78. El Fuero de los Españoles contemplaba este derecho sólo para los trabajadores. Aún hoy no todos tienen de facto el derecho a la protección de la salud.

(6) Véase: El precio de la salud y el Coste de la Enfermedad. Serie de Monografías, n 19. Ginegra 19.

(7) Salud Mundial. Junio 1979. Esa es la perspectiva con la que E. Najera aborda la teoria de la Sanidad en "Conceptos actuales y tendencias del concepto de Sanidad", Rev. San. Hig. Púb. LI; 5-6. Pp. 639-654 y "Teoría general de la Salud Pública", Rev. San. Hig. Púb. LII; 1-2, pp. 65-79.

(8) Emitida en el Congreso de Médicos y Bíblogos de Lengua Catalana. 1981. 
terapéutica hasta que la revolución farmacológica permitió actuar con éxito frente a la enfermedad, planeó en la evolución de los conceptos desde Sanitas (Sanidad) a Salud Pública. Lo importante, lo decisivo, era la praxis y la praxis eficaz por lo que en su origen Salud Pública. Public Health o Sante Publique fue el conjunto de medidas tomadas por el Estado para conquistar las zonas ocupadas por las enfermedades infecciosas, preocupación primera en los tiempos originarios. ${ }^{9}$

Desde este momento la salud pública estaba caracterizada por un elemento negativo y dos positivos. La salud pública es lo contrario a la salud privada; salud pública es positivamente, prevención y obligación. En términos generales lo que ha permanecido en manos privadas han sido los cuidados médicos, únicos generadores de plusvalía hasta hace pocos, escasos años. Salud Pública sería desde este punto de vista lo que no es salud privada, además, como ya va dicho, de todos los mecanismos preventivos y de todas las obligaciones (vacunas, cuarentenas, registros, certificaciones, ...)

Cercano a este pensamiento originario esta $\mathrm{M}$. Terris ${ }^{10}$ quien por la vía de los programas a poner en marcha, por vía de nuevo de la praxis, desarrolla la salud pública a través de las grandes tareas a realizar hoy por ella: el control del medio ambiente, los exámenes de detección masiva y la educación para la salud. Ahora bien $\mathrm{M}$. Terris no se limita a estas acciones, la realidad de la imposible cobertura individual del riesgo y pago de la enfermedad-crónica-invalidante se impone en la mente de este autor, y de todos los estudiosos, quien incorpora a las tareas actuales y futuras de la salud pública, la de "hacer desaparecer las diferencias que existen para el acceso a la atención médica"."

Hoy desde Lalonde ${ }^{12}$ hablando de campo de la salud para indicar el marco en el cual aquella se genera, se pierde, o se recupera. La biología humana como potencialidad máxima y limitativa cuya manipulación es escasa, el medio ambiente del que depende para la salud o la enfermedad el $90 \%$ de las posibilidades, el modo de vida patógena saludable ${ }^{13}$ y los cuidados médicos son el marco o campo de la salud. En el se engloban las actividades prácticas que pueden ser establecidas para buscar una mejor salud, medidas que son dirigidas hacia los individuos y las colectividades, libremente asumidas o coercitivamente dictadas por quien representa la voluntad popular, medidas en último término de salud pública.

Según la opinión vulgar, la ciencia de la medicina ha sido la funete de todos los progresos realizados en el campo de la salud, y.la mayor parte considera el nivel de

(9) Estamos en 1980.

(10) M. Terris. Las grandes tareas de la Salud Pública en "La Revoluciớn epiderniológica". Siglo XXI, Madrid, 1980.

(11) M. Terris, o.c. pp. 89 y ss. En el mismo sentido se pronuncia T. Mekeown en "El papel de la medicina". Siglo XXI. Madrid. 1982 y en "Introducción a la medicina social". Siglo XXI. Madrid. 1981. C. Berlinguer en "Medicina y Polfíca". Edit. Cuano Mundo. Buenos Aires. 1975. Y A. Neri en "Salud y política social". Nachette. Buenos Aires. 1983.

(12) M. Lalonde, Nouvelle perspective de la Santé des canadiens. Gobiemo de Canada. Otawa. 1974.

(13) Hoy hablamos de "enfermedades buscadas" o elegidas para significar que el modo de vida es fuente u origen voluntario de enfermedades venéreas, las ligadas al hábito de fumar o los accidentes de los deportistas. 
salud y la calidad de la medicina como sinónimo. Para el conjunto de la población, el dominio de la salud y el sistema de cuidados médicos es equivalente. Esta imagen ha sido reforzada por la publicidad sobre los productos farmacéuticos y otros instrumentos de la acción médico quirúrgica, así como las telenovelas que presentan al médico como $\mathrm{a}$ un héroe y por los sentimientos edipianos que el médico suscita en sus pacientes. Por estas razones, la mayor parte de los gastos directos en salud giran alrededor del médico particularmente las consultas, los hospitales, los gastos de laboratorio y la prescripción de medicamentos. El $80 \%$ de los gastos de nuestro sistema radica en el tratamiento de los enfermos.

Sin embargo, toda esta concepción tradicional quiebra cuando estudiamos las causas de enfermedad y muerte de los seres humanos. Las tasas de morbilidad y mortalidad demuestran sin lugar a dudas que el medio ambiente insalubre, las formas de vida patógenas, los condicionamientos limitantes de la biología y el hambre pura y simple son los responsables de las enfermedades infecciosas, las cardiovasculares, la diabetes, los accidentes o las enfermedades mentales grandes matadoras o fuente de sufrimientos para nuestros conciudadanos. ${ }^{14}$

Veamos esto con detalle: la biología humana engloba todos los aspectos de la salud que tiene su origen en el interior del organismo o dependen de la estructura biológica, provocan innumerables sufrimientos y cuentan muchos miles de pesetas, lo cierto es que están todavía por investigar en toda su profundidad. El medio ambiente represente el conjunto de factores exteriores al cuerpo que tienen una incidencia sobre la salud y escapan al dominio individual: los alimentos, el agua, el aire; el ruido, el medio social y sus transformaciones, tienen repercusiones nefastas para la salud y son razón suficiente del noventa por ciento de las enfermedades, respecto del medio las acciones más frecuentes son más degradar que mejorar. La forma de vida representa el conjunto de decisiones que tomamos individualmente y que en nuestro caso tienen que ver con la salud. El comportamiento y la conducta que dafian la salud, crean riesgos innecesarios a los cuales las personas nos exponemos deliberadamente, de tal modo que podemos afirmar que nuestra propia forma de vida es causa directa o indirecta de nuestra propia muerte. Las enfermedades así elegidas, ocupan los primeros lugares de las tablas de mortalidad: crisis cardíacas, cáncer de pulmón, o bronquitis son elegidas por los que hacen del tabaco su droga blanda, al igual que la cirrosis o el accidente de tráfico son elegidos por los que hacen del alcohol su droga dura. Lo cierto es que poco hacemos para modificar individual o socialmente nuestro modo de vida.

A última hora, el cuarto elemento constitutivo de lo que hemos llamado junto con la biología, el medio ambiente y la forma de vida, campo o marco de la salud, es decir la organización de los cuidados sanitarios, médicos y de enfermería si hubiera que hacer tal distinción, que comprende todo lo que alrededor de la curación de la enfermedad hay, es de los cuatro elementos al menos importante en cuanto generador de salud, y sin embargo el que más recursos exige y obtiene. La mayor parte de los esfuerzos desplegados hasta aqui por la sociedad para mejorar la salud, han sido en el sector de los cuidados sanitarios, mientras que las principales causas de muerte y enfermedad tienen sus raices en los tres primeros elementos. Es evidente que sumas con-

(14) De esta Ley General no se excluyen los ciudadanos españoles. 
siderables de dinero se han gastado para tratar enfermedades que no tenían que haber existido.

Como estos criterios, criterios científicos, hemos de dar un paso adelante, paso simple en su enunciación, aunque difícil y complejo en su realización, pues de lo que se trata es ni más ni menos que de cambiar nuestro sistema sanitario y como Copérnico situar en el centro la salud y en la periferia la enfermedad, y que en lo que a la enseñanza se refiere, significa elaborar un currículum escolar en el que la salud sea el centro de nuestra preocupación. Se trata de comenzar la revolución epidemiológica enseñando a los participantes en nuestras actividades docentes, que la ciencia empieza a influir en la toma de decisiones referentes a su formación.

\section{Salud Pública: concepción personal}

Establecido que la salud es un bien de los individuos y de las colectividades distinto de la no enfermedad y que se inscribe en una curva cuyo límite está en el infinito, no nos costará trabajo afirmar en una primera aproximación con Winslow que salud pública es "la ciencia y el arte de impedir la enfermedad, prolongar la vida y fomentar la salud mental y física y la eficacia a través de los esfuerzos organizados de la comunidad para el saneamiento del medio ambiente, el control de las infecciones trasmisibles, la educación del individuo en higiene personal, la organización de servicios médicos y de enfermería para el diagnóstico precoz y el tratamiento preventivo de la enfermedad y el desarrollo de una organización social que asegure a cada individuo un nivel de vida adecuado para el mantenimiento de la salud, organizando estas prestaciones de forma que permita que cada ciudadano lleve a la práctica su derecho innato a la salud y a la longevidad". ${ }^{15}$

En esta definición están implícitos, como han visto certeramente Clavero y Sierra ${ }^{16}$ la naturaleza de la Salud Pública, ciencia y arte, sus funciones, la exigencia de una organización y los objetivos de prevención de la enfermedad y promoción de la vida. A partir de aquí todas las definiciones son la profundización o actualización de uno u otro de sus componentes, ${ }^{17}$ por cuanto Winslow destaca el espíritu progresista de la Salud Pública, aun cuando concrete en demasía la definición a las actividades propias de su época.

Lo que hace difícil definir Salud Pública es el continuo cambio a que está sometida, por ello es preferible distinguir en la noción que nos ocupa tres niveles o planos de análisis: uno primero a nuestra vista es el que se identifica con las actividades sanitarias

(15) C.E.A. Winslow, The unfilled field of Public Health. Mod. Med. 2: 183, 1920.

(16) G. Clavero y A. Sierra, Actualización del concepto de Salud Pública. Primera Ponencia de las II Jomadas Técnicas del Cuerpo Médico de Sanidad Nacional. Rev. San. Hig. Púb. 57, 221-254. 1983.

(17) La bibliografía sobre Salud Pública y su evolución es amplia y extensa, cito solamente a efectos orientativos a W. Hobson. The Theory and practice of Public Health, Oxford U.P. N. York. 1965; J.I. Hanlon. Public Healt. Administración and practice. Mosby. St. Louis. 1974. R. E. Freenan y E. M. Holnes, Administración de los Servicios de Salud Pública. Interamerica. Madrid. 1963; N. San Martín, Sante Publique et medicine preventive. Masson. Paris. 1983; C. A. Alvarado, Concepto de Salud Pública en Medicina Sanitaria. A. Sonis edit. El Ateneo. Barcelona. 1982. 
puestas en marcha en un momento histórico otro plano más profundo es el que se percibe en la definición de Winslow; finalmente un último plano es que nos aparece cuando consideramos que la búsqueda de la salud se identifica con la persecución del bienestar. Este último plano es más bien una declaración de principios, un objetivo en un límite inalcanzable pero cuya persecución es de exigencia inexorable. El plano intermedio es el que nos permitirá estudiar la salud pública dentro de los límites de "lo que tiene que ver directamente con la promición, prevención y restauración de la salud". El nivel más superficial nos facilitará hacer la lista de las actividades que hoy día se utilizan para conseguir la salud de la colectividad.

Cualquiera de estos niveles de realidad puede servirnos de brújula para orientar nuestros ańlisis, pero si utilizamos el último podríamos caer en la confusión de identificar con lo sanitario, y en último término con lo médico, todas las acciones que pueden arbitarse para conseguir el bienestar. En este sentido hemos aceptado las tesis de Gremy y Pissarro cuando afirman que una política de promoción de la salud es "el objetivo fundamental, no ya de la política sanitaria, sino de una sociedad democrática". ${ }^{18}$

Por el contrario el primer plano del análisis no debe servirnos en ningún momento como materia de reflexión, pues podría llevarnos a identificar la sanidad con las funciones y organización del Ministerio adjetivado con su nombre. Ahora bien, un análisis de organización, que por necesidad es algo concreto, no puede situarse en la intemporalidad, por lo que será el segundo plano, intermedio, el que concluirá nuestro análisis, teniendo en cuenta que tal organización es comparable a un ejército en movimiento cuyos efectivos, disposición y estrategia ha de variar de acuerdo a los problemas que hemos de vencer, olvidando felizmente a los enemigos que hayamos vencido. Así nuestro análisis tendrá que ver con las actividades que se relacionan directamente con la promoción, mantenimiento y restauración de la salud. ${ }^{19}$

(18) F. Gremy y B. Pissarro, Proposition pour une politique de prevention. Informe al Ministro de Sanidad. Marzo. 1981, p. 61.

(19) Esta es a mi modo de ver la posición que adoptan Clavero y Sierra cuando analizan las ciencias que participan o han participado en el desarrollo de la Salud Pública. 
LA EVOLUCION DE LA CIENCIA MEDICA HASTA EL AÑO 2000

Desde la era empírica hasta la era de la salud entendida como ciencia política

\begin{tabular}{|c|c|c|}
\hline & Era Empírica & $\begin{array}{c}\text { Era de las } \\
\text { Ciencias Básicas } \\
\end{array}$ \\
\hline \multirow[b]{2}{*}{ Principios y fines } & $\begin{array}{l}1850 \\
\text { Orientación Sintomática }\end{array}$ & $\begin{array}{c}1900 \\
\text { Orientación Bacteriológica o } \\
\text { Patológica }\end{array}$ \\
\hline & $\begin{array}{l}\text { Diagnóstico y tratamiento } \\
\text { pírico de los sintomas }\end{array}$ & $\begin{array}{l}\text { Diagnóstico y tratamiento de } \\
\text { las enfermedades }\end{array}$ \\
\hline Enseñanza & $\begin{array}{l}\text { Lecciones magistrales. } \\
\text { Intruscción autoritaria }\end{array}$ & $\begin{array}{l}\text { Enseñanza } \\
\text { de laboratorio }\end{array}$ \\
\hline Investigación & Histórica & $\begin{array}{l}\text { Básica de laboratorio. } \\
\text { Perfeccionamiento de } \\
\text { nuevos médicos }\end{array}$ \\
\hline \multirow[t]{2}{*}{$\begin{array}{l}\text { Ciencias del } \\
\text { comportamiento }\end{array}$} & Desconocidas & Innecesarias \\
\hline & & Actividad individual \\
\hline & & \\
\hline
\end{tabular}


Cont. LA EVOLUCION DE LA CIENCIA MEDICA HASTA EL AÑO 2000

Desde la era empírica hasta la era de la salud entendida como ciencia política

\begin{tabular}{|c|c|c|}
\hline $\begin{array}{l}\text { Era de las ciencias } \\
\text { clínicas }\end{array}$ & $\begin{array}{l}\text { Era de la } \\
\text { Salud Pública }\end{array}$ & $\begin{array}{c}\text { Era de la } \\
\text { Ciencia Política }\end{array}$ \\
\hline $\begin{array}{l}1950 \\
\text { Orientación Individual }\end{array}$ & $\begin{array}{c}1975 \\
\text { Orientación Comunal }\end{array}$ & $\begin{array}{l}2000 \\
\text { Orientación a la } \\
\text { población }\end{array}$ \\
\hline $\begin{array}{l}\text { Diagnóstico y } \\
\text { tratamiento de casos } \\
\text { individuales }\end{array}$ & $\begin{array}{l}\text { Diagnóstico y tratamiento } \\
\text { de enfermedades de la } \\
\text { comunidad }\end{array}$ & $\begin{array}{l}\text { Diagnóstico y tratamiento } \\
\text { de enfermedades de todo el } \\
\text { cuerpo político }\end{array}$ \\
\hline $\begin{array}{l}\text { Instrucción clínica. } \\
\text { Aprendizaje "a la } \\
\text { cabecera del } \\
\text { enfermo". }\end{array}$ & $\begin{array}{l}\text { Instrucción clínica en salud } \\
\text { pública. Aprendizaje en } \\
\text { comunidad. }\end{array}$ & $\begin{array}{c}\text { Experiencia social. } \\
\text { Conocimientos de economía } \\
\text { y sociologia. Aptitudes de } \\
\text { gestión. Psicología política } \\
\text { y procesos políticos. } \\
\text { Programa nacional. }\end{array}$ \\
\hline $\begin{array}{l}\text { Perfeccionamiento } \\
\text { de técnicas clínicas }\end{array}$ & $\begin{array}{l}\text { Comunitaria. De } \\
\text { desarrollo de la } \\
\text { comunidad. De } \\
\text { indices y criterios } \\
\text { de técnicas de } \\
\text { planificación. }\end{array}$ & $\begin{array}{c}\text { BIndices sociales y } \\
\text { económicos de desarrollo de } \\
\text { la salud. Indices subjetivos } \\
\text { de calidad de vida. Procesos } \\
\text { intersectoriales. Procesos en } \\
\text { redes. }\end{array}$ \\
\hline Secundarias & Integradas & Interrelacionadas \\
\hline $\begin{array}{c}\text { Ciencias sociales auxiliares } \\
\text { de la Medicina. }\end{array}$ & $\begin{array}{l}\text { Ciencias sociales. Aptitud } \\
\text { muy compleja. Igualdad con } \\
\text { la ciencia de la salud }\end{array}$ & $\begin{array}{c}\text { Ciencias sociales. Ciencias de } \\
\text { la salud. Ciencias políticas y } \\
\text { económicas. }\end{array}$ \\
\hline Necesidad individual & Equipos interdisciplinarios & Equipos intersectoriales \\
\hline
\end{tabular}




\section{Esfera de acción de la salud pública}

Después de lo dicho sobre la definición, procede catalogar las actividades que deben ser utilizadas para conseguir el último objetivo de la Salud Pública. De acuerdo con los adelantos técnicos y científicos, esta esfera de acción ha pasado desde sus humildes comienzos, reducida a las formas más rudimentarias del saneamiento ambiental, hasta la utilización de los ordenadores con fines organizativos, pasando por las llamadas formas positivas de la higiene personal encaminadas al fomento de la salud. Estas actividades pueden agruparse en cinco grandes epígrafes:

a) Control de medio físico. Su fin inmediato estará orientado a evitar los daños a la salud procedentes del medio físico $y$, en un espíritu más progresivo a suministrar un medio que favorezca el crecimiento de la misma.

b) Considerando la patología en su sentido más amplio, la prevención de la enfermedad llena este epígrafe. La prevención de las enfermedades infecciosas ha sido históricamente la razón de ser de la sanidad. Hoy esta campo se ha ensanchado y abarca no sólo éstas sino los accidentes, las enfermedades crónicas y las mentales. ${ }^{20}$

c) Restauración de la salud perdida. El hospital como prototipo agrupa a su alrededor las actividades curativas.

d) Entendiendo que la capacidad genética puede y debe desarrollarse al máximo, la esfera de acción de la salud pública en la promoción de la salud individual y colectiva proyecta a horizontes nuevos las antiguas prácticas de higiene.

e) Organización de las anteriores actividades. La complejidad de medios, personales y materiales, empleados en las tareas sanitarias, justifican un epígrafe integrador de todas las que intentan su eficacia y eficiencia.

\section{Actividades que buscan controlar el medio físico}

Las actividades que podemos denominar de saneamiento son las básicas en un programa sanitario. Lo fueron históricamente, recuérdese las obras públicas romanas y lo serán en el futuro, protección contra las radiaciones ionizantes. Con un sistema de saneamiento adecuado desaparecerian el $90 \%$ de las enfermedades mundiales, lo que es más dudoso es que las actividades necesarias sean estrictamente médicas o deban estar dirigidas por médicos. Entre tales actividades se encuentran:

* Abastecimiento de agua potable.

* Eliminación de basuras y aguas residuales.

* Tratamiento y depuración.

* Protección de cursos de agua, playas y costas.

(20) El adelanto médico más importante del siglo XIX fue el descubrimiento de que las enfermedades infecciosas son atribuibles en gran parte a las condiciones ambientales y con frecuencia, pueden prevenirse mediante el control de las influencias de las que se derivan; el adelanto más considerable del siglo XX es el reconocimiento de lo mismo, cabe decir de muchas enfermedades transmisibles. Del Informe de la Primera Reunión del Subcomilé del CCIM sobre Estrategia de la Investigación Sanitaria para la SPT/2000. Crónica de la OMS, 39; 2: $73-78$ (1985). 
* Control de la contaminación atmosférica.

* Protección contra las radiaciones ionizantes.

* Urbanismo e higiene de las viviendas.

* Eliminación de roedores y otros vectores de enfermedad.

* Saneamiento de los medios de transporte.

* Saneamiento ganadero.

* Supervisión y control higiénico de los alimentos.

* Seguridad vial.

* Policía sanitaria mortuoria.

* Control de las actividades molestas, insalubres, nocivas y peligrosas.

* Higiene industrial y de los medios de trabajo.

Tal vez de esta lista pueda eliminarse algún componente en el futuro, pero lo más seguro es que nuevos riesgos se incorporen. La complejidad es, y será, tanta que algunos paises han establecido el título de ingeniero sanitario para denominar al técnico de grado superior especialista en este campo. De nuevo hay que insistir aqui que las acciones de saneamiento constituyen la base de una política sanitaria de progreso y que la mayor parte de las mismas escapan de la esfera médica.

\section{Actividades sanitarias preventivas}

La prevención de las enfermedades infecciosas ha sido la razón de ser de la salud pública; hoy el campo de la prevención se ha ensanchado y abarca todos los supuestos de posibilidad de pérdida de la salud, sin embargo no todas las acciones preventivas son acciones médicas particularmente, las más eficaces por intentar remover las causas de la enfermedad. ${ }^{21}$

Entre las afecciones que se benefician de la prevención se encuentran:

* Enfermedades transmisibles.

* Enfermedades cardiovasculares.

* Enfermedades reumáticas.

* Cánceres.

* Afecciones sensoriales y hereditarias.

* Reumatismos.

* Enfermedades profesionales y del trabajo.

* Enfermedades mentales.

* Accidentes de trabajo, domésticos y profesionales.

* Enfermedades carenciales y de la nutrición.

* Malformaciones congénitas.

Si se desea podemos clasificar todas las enfermedades con una perspectiva operacional, tal y como ha hecho el Comité Consultivo de Investigaciones Médicas

(21) Estas ideas se desarrollarán extensamente cuando tratemos del concepto y contenido de la Medicina Preventiva. 
Mundial atendiendo no solamente a los tradicionales mecanismos fisiológicos o patológicos sino en función de sus origenes. ${ }^{22}$ La primera categoria incluirá las enfermedades determinadas antes del nacimiento, ya sea en la fecundación o en el curso de la vida intrauterina. La segunda las enfermedades debidas a problemas de adaptación.

La desaparición de un buen número de entidades patológicas que en otros tiempos formaban parte de esta lista son el exponente de la eficacia de la prevención.

\section{Actividades curativas y otras con ellas vinculadas}

Los centros curativos, y el hospital como prototipo, llenan este capítulo de la acción sanitaria. Alrededor del hospital se articulan una serie de actividades cuya finalidad es devolver a la comunidad en condiciones óptimas al enfermo ya curado o en las mayores capacidades residuales posibles. Entre ellas se encuentran las siguientes:

* Servicios de enfermería.

* Servicios de cuidados a domicilio.

* Servicios de rehabilitación.

* Política de farmacia.

* Servicios de ambulancias.

* Servicios para enfermedades de larga duración.

\section{Actividades de promoción de la salud individual y colectiva}

La salud como bien es algo que puede mejorarse. Las antiguas prácticas de higiene individual alcanzan así su máxima proyección y se incrementan con otras similares. En verdad, partiendo de la definición de salud positiva, como plenitud de vida, armonía del hombre consigo mismo y con su medio de tal modo que le permita sacar el máximo partido a sus capacidades, afirmamos que la promoción de la salud es algo más, bastante más, que una actividad médica o sanitaria, para convertirse en el criterio más certero para juzgar la progresiva de la labor de los gobiemos. ${ }^{23}$ En la esfera que nos ocupa son actividades promotoras de la salud:

* Protección maternal e infantil.

* Higiene de la edad preescolar.

* Educación física y deporte.

* Alimentación y nutrición.

* Exámenes periódicos de salud.

* Salud mental.

* Educación sanitaria.

* Higiene profesional.

* Gerocultura.

(22) Informe de la Primera Reunión... p. 15.

(23) Propositions pour une politique... p. 54. 
El paulatino aumento de estas actividades es lo que caracteriza a la sanidad progresiva.

\section{Actividades de coordinación; organización y control}

La complejidad de medios empleados en las tareas sanitarias, justifican que dentro de su campo de acción, incluyamos las que intentan unificarlas, rentabilizarlas y hacerlas más eficaces. Entre ellas:

* Vigilancia epidemiológica.

* Estadísticas demográficas y sanitarias.

* Formación y perfeccionamiento del personal.

* Investigación básica y aplicada.

A la vista de lo anterior no es extraña la afirmación que Clavero y Sierra hacen, acerca de la necesidad que la Salud Pública ha tenido de asumir e integrar parcelas de conocimiento procedentes de numerosas ciencias tanto biológicas como sociales, ${ }^{24}$ afirmación que tendrá reflejo en la variedad de materias, ciencias y temas que habrá de conocer el participante en nuestras actividades docentes de un modo $u$ otro. Los autores mencionados citan cuatro conjuntos de ciencias fundamentales.

* Ciencias de la naturaleza: Física, química y biología.

* Ciencias sociales: Derecho, economía, sociología, política, demografía, sicología y antropología.

* Ciencias mixtas o humanas: Ecología, epidemiología y medicina.

* Ciencias instrumentales de la salud pública: Estadística, informática, administración, ingeniería, farmacia, arquitectura, pedagogía.

La salud pública, en fin, "es la resultante de la permanente integración de los conocimientos de numerosas ciencias aplicadas a un objetivo común: el bienestar físico, síquico y social de la comunidad. Como práctica es una actividad multiprofesional especializada en la aplicación de esa teoría y encuadrada en el seno de la Administración Pública". ${ }^{25}$

(24) Actualización del concepto... pp. 225 y ss.

(25) Actualización del concepto... pp. 225 y ss. 